\title{
Einfluss von „Home Numeracy Environment“ auf die mathematische Kompetenzentwicklung vom Vorschulalter bis Ende des 1. Schuljahres
}

\author{
The impact of Home Numeracy Environment on the development of \\ mathematical competencies from pre-school until the end of Grade 1
}

\begin{abstract}
Zusammenfassung:
Mathematische Kompetenzen sind nicht nur für schulischen Erfolg, sondern auch später für viele Berufe wichtig. Während zahlreiche Untersuchungen zur sprachlichen Kompetenzentwicklung und deren Zusammenhang mit der familiären Lernumwelt vorliegen, wurde der Bereich Mathematik bislang nur randständig untersucht. Dabei weisen ersten Studien auf die Bedeutsamkeit der mathematischen Lernumwelt in Familien, der sogenannten „Home Numeracy Environment“ (HNE), für die mathematische Kompetenzentwicklung hin. Befunde der vorliegenden Längsschnittstudie mit etwa 600 Kindern vom Kindergarten bis Ende der ersten Klasse zeigen den Einfluss mathematischer Aktivitäten der Eltern wie z.B. Würfel- oder Zahlenspiele auf die mathematischen Fähigkeiten der Kinder. Die positiven Effekte bleiben auch unter Kontrolle der Intelligenz der Kinder bestehen. Weiterhin sagt die HNE nicht nur die mathematischen Kompetenzen beim Schuleintritt vorher, sondern beeinflusst darüber hinaus auch die weitere mathematische Kompetenzentwicklung. Die Befunde werden in einem Strukturgleichungsmodell dargestellt und hinsichtlich ihrer praktischen Implikationen diskutiert.
\end{abstract}

Schlagwörter: Home Numeracy Environment, mathematische Kompetenzentwicklung, familiäre Lernumwelt, Würfel- und Zahlenspiele, Kindergarten- und Schulkinder

\begin{abstract}
:
Mathematical competencies are important not only for academic achievement at school, but also for several professions later in life. Although plenty of research has focused on the development of linguistic competencies and the impact of learning environments in families, mathematics has mostly been neglected so far. However, a few initial studies indicate that the mathematical learning environment in families, the so-called "Home Numeracy Environment" (HNE), is important for the development of mathematical abilities. The present longitudinal study was carried out from kindergarten until the end of Grade 1, using a sample of about 600 children. Results show the impact of family-related mathematical activities, such as playing dice or number games, on the development of mathematical competencies. The positive effects remained even after intelligence had been controlled for. Moreover, HNE was not only a significant predictor of mathematical abilities at the end of kindergarten, but it also influenced the further development of mathematical competencies above and beyond its initial impact. Results are shown in a structural equation model, and are discussed with regard to their practical implications.
\end{abstract}

Key words: Home Numeracy Environment, development of mathematical competencies, learning environments in families, dicing and number games, preschool and school children 
Mathematische Kompetenzen spielen nicht nur für die erfolgreiche Bewältigung der Schullaufbahn, sondern auch im Alltag sowie im späteren Berufsleben eine große Rolle (vgl. Duncan et al. 2007; Geary 2000). Deshalb ist es wichtig, Kinder beim Erwerb mathematischer Fähigkeiten frühzeitig zu unterstützen. Die Grundlage guter mathematischer Kompetenzen wird schon sehr früh in der kindlichen Entwicklung geschaffen, und mathematische Leistungen zu Schulbeginn gehören zu den besten Prädiktoren für spätere Schulleistungen (Claessens et al. 2009; Krajewski/Schneider 2009; Stern 2009).

Mathematisches Lernen beginnt dabei schon vor der Einschulung, und bereits im ersten Lebensjahr weisen Kleinkinder bestimmte mathematische Kompetenzen auf (vgl. Butterworth 2005; Resnick 1989). In der Münchner Längsschnittstudie LOGIK (Longitudinalstudie zur Genese individueller Kompetenzen) zeigte sich, dass numerische Kompetenzen im Alter von fünf Jahren einen mittelhohen Zusammenhang $(r=.43)$ mit der mathematischen Intelligenz im Alter von 23 Jahren aufwiesen (Stern 2008). Die hohe Vorhersageleistung über diesen langen Zeitraum verdeutlicht die Bedeutung früher mathematischer Fähigkeiten.

Mittlerweile wurden verschiedene Aspekte von informellem mathematischem Wissen bei Kindergartenkindern identifiziert, welche spätere mathematische Schulfähigkeiten gut vorhersagen können. Insbesondere das frühe Wissen über Mengen und Zahlen sowie deren Relation zueinander scheinen von großer Bedeutung zu sein (Geary 2011; Krajewski/Schneider 2009). Diese frühen Kompetenzen sind sehr spezifische Prädiktoren für mathematische Leistungen, auch wenn allgemeinere Fähigkeiten wie beispielsweise die Intelligenz berücksichtigt werden. Dennoch sollte auch die Bedeutung der Intelligenz nicht unterschätzt werden. Selbst als eher unspezifischer Prädiktor kann sie die Varianz mathematischer Schulleistungen bedeutsam aufklären (vgl. Hasselhorn/Gold 2009).

Neben den individuellen Eigenschaften eines Kindes spielt jedoch auch die Lernumwelt eine wichtige Rolle. Neben Aspekten wie beispielsweise dem Kindergartenbesuch (vgl. Roßbach 2005; Walston/West 2004) übt in frühen Jahren insbesondere der Lernkontext Familie einen großen Einfluss auf die Kompetenzentwicklung von Kindern aus. So beschreiben Brunner und Noack (2010) die Familie als wichtigen Kontext sowohl für die gesunde emotionale Entwicklung von Kindern und Jugendlichen als auch als Voraussetzung für eine optimale intellektuelle Entwicklung. Auch Helmke und Schrader (2010) betonen die wichtige Rolle der familiären Lernumwelt für schulische Leistungen neben anderen Faktoren wie Schule, Gleichaltrige und Persönlichkeitsmerkmale.

Mittlerweile liegen viele Studien zur sogenannten „Home Literacy Environment“ (HLE) vor, welche sprachliche Leistungen von Kindern gut vorhersagen kann (z.B. Hood et al. 2008; Rashid et al. 2005). Dieses Konstrukt umfasst dabei diejenigen familiären Aspekte, die Einfluss auf sprachliche und schriftsprachliche Kompetenzen von Kindern nehmen (Niklas/Schneider 2010; vgl. auch Lehrl et al. in diesem Band). So sind beispielsweise das Leseverhalten der Eltern, die Häufigkeit des Vorlesens oder auch die Anzahl an Büchern im Haushalt eng mit den späteren Lesefähigkeiten der Kinder verknüpft.

Während die Bedeutung von HLE für die Sprach- und Schriftsprachentwicklung bereits gut belegt ist, ist der Zusammenhang mit mathematischen Kompetenzen bislang nicht endgültig geklärt. Nach Whitehead (2004) sind Aspekte von HLE wie das gemeinsame Betrachten von Bilderbüchern und das Vorlesen nicht nur für das Lesen, sondern auch für andere schulische Bereiche von Bedeutung. Anders zeigte sich dies in einer Untersuchung von Niklas und Schneider (2010), die zwar eine signifikante Korrelation zwi- 
schen HLE und frühem Mengen-Zahlenwissen im Alter von fünf Jahren belegen konnten. Dennoch klärte hier die HLE unter Einbezug von Kontrollvariablen, wie z.B. der Intelligenz, weniger als 1\% zusätzliche Varianz der mathematischen Vorläuferfertigkeiten auf. Auch in anderen Studien erwies sich HLE als spezifischer Prädiktor für sprachliche, nicht aber für mathematische Kompetenzen (z.B. Christian et al. 1998).

Wird das Konzept der familiären Lernumwelt jedoch erweitert, ändert sich auch diese Spezifität. So fassten Melhuish und Kollegen (2008) unter ihrem Konstrukt „Home Learning Environment" sowohl sprachliche Aktivitäten (z.B. Vorlesehäufigkeit) als auch mathematische Aktivitäten (z.B. Häufigkeit von Zahlenspielen) in der Familie zusammen. Dieses Konstrukt erwies sich in ihren Analysen als signifikanter Prädiktor sowohl für sprachliche als auch für mathematische Kompetenzen der Kinder. Es ist also zu erwarten, dass sich spezifische Zusammenhänge mit mathematischen Fähigkeiten eher für Aspekte der Lernumwelt mit mathematischem Kontext finden lassen sollten.

Mathematikspezifische familiäre Lernumwelten werden als „Home Numeracy Environment" (HNE) bezeichnet. In Analogie zu HLE umfasst das Konstrukt HNE dabei ganz allgemein diejenigen Aspekte der familiären Lernumwelt, welche die mathematischen Fähigkeiten der in dieser Lernumwelt lebenden Kinder beeinflussen sollten. Bei diesen Aspekten kann es sich um Aktivitäten in der Familie handeln, die in irgendeiner Form Bezug zu mathematischen Inhalten haben (z.B. das Spielen von Würfel-, Rechen- oder Zahlenspielen). Hinzugerechnet werden kann auch der „mathematische Besitz“ einer Familie bzw. eines Kindes (z.B. Uhr, Taschenrechner, Lineal oder Maßband im Kinderzimmer). Weiterhin spielen das Modellverhalten und somit die mathematikbezogenen Einstellungen und Verhaltensweisen der Eltern eine Rolle (vgl. z.B. Ehmke/Siegle 2008).

LeFevre und Kollegen (2009) konnten zeigen, dass die Häufigkeit, mit der indirekt mit Zahlen verbundene Spiele (z.B. Würfelspiele) in der Familie gespielt wurden, schwach, aber bedeutsam mit den mathematischen Kompetenzen der Kinder bei Schulanfang zusammenhängt (vgl. auch Blevins-Knabe/Musun-Miller 1996). Grundlage hierfür ist, dass selbst bei relativ basalen Aktivitäten wie Karten-, Brett- und Würfelspielen mathematische Strukturen deutlich gemacht werden, was dann wiederum zur Förderung mathematischer Fähigkeiten beiträgt (Werner 2009).

In Bezug auf HNE sollte allerdings zwischen direkten und indirekten Lernaktivitäten unterschieden werden (LeFevre et al. 2009). Während indirektes mathematisches Lernen beispielsweise beim Würfelspiel auftritt, findet formelles und direktes mathematisches Lernen in der Familie statt, wenn Eltern ihren Kindern gezielt das Zählen oder Rechnen beibringen. Es finden sich sowohl Studien, die die Bedeutung früher direkter Aktivitäten in der familiären Lernumwelt betonen (z.B. Huntsinger et al. 2000; LeFevre et al. 2002), als auch Forscher, die eher den spielerischen Kontext in den Vordergrund rücken (z.B. Stern 2006).

Insgesamt ist davon auszugehen, dass beide Aspekte, also die direkte und indirekte Förderung mathematischer Fähigkeiten in der Familie, eine Rolle spielen, wobei noch nicht endgültig geklärt ist, welche Komponente wichtiger für die Kompetenzentwicklung ist (vgl. LeFevre et al. 2009; LeFevre et al. 2010).

Bislang messen sowohl Eltern als auch Erzieher der mathematischen gegenüber der schriftsprachlichen Kompetenzentwicklung einen geringeren Wert bei, obwohl mathematische Fähigkeiten sehr wichtig für die Bildungslaufbahn sind (Niklas 2011; vgl. auch Blevins-Knabe et al. 2000; LeFevre et al. 2009; Skwarchuk 2009). Zudem bieten gerade 
Eltern mit geringeren mathematischen Kompetenzen oder Selbstvertrauen häufig eine schlechtere Unterstützung für die mathematische Kompetenzentwicklung ihrer Kinder (Ehmke/Siegle 2008; Hyde et al. 2006; Skwarchuk 2009). Sollte aber eine höherwertige HNE, bestehend aus einer höheren Frequenz an einfachen mathematischen Spielen im Vorschulalter, zu besseren mathematischen Fähigkeiten bei den in dieser Lernumwelt lebenden Kindern führen, böte sich hier ein Ansatz, der für alle Eltern geeignet erscheint, um ihre Kinder in deren mathematischer Kompetenzentwicklung besser zu unterstützen.

\section{Fragestellung}

Bislang sind kaum Untersuchungen zu HNE im deutschsprachigen Raum vorhanden. Da aber erste Befunde darauf hinweisen, dass informelle und formelle mathematische Aktivitäten in der Familie Kinder beim Erwerb mathematischer Fähigkeiten unterstützen können, soll die vorliegende Studie dies anhand einer größeren deutschen Stichprobe überprüfen. Die HNE wurde dabei über einen Elternfragebogen erfasst, in dem mathematische Interaktionen zwischen Eltern und Kind erfragt wurden. Die Fragen bezogen sich dabei auf die Häufigkeit, mit der Würfel-, Rechen- und Zahlenspiele gespielt wurden.

Konkret soll überprüft werden, ob eine besser ausgeprägte HNE mit besseren mathematischen Fähigkeiten vor Schuleintritt zusammenhängt und ob sich darüber hinaus zusätzliche Effekte auf die weitere mathematische Kompetenzentwicklung unter Kontrolle kognitiver Ausgangsunterschiede zeigen. Es wird dabei erwartet, dass mathematische Aktivitäten in der Familie als Prozessmerkmale direkt die mathematischen Kompetenzen der Kinder beeinflussen. Dies sollte auch der Fall sein, wenn die Intelligenz der Kinder sowie ihre frühen mathematischen Kompetenzen im Kindergarten berücksichtigt werden. Abbildung 1 zeigt das zu untersuchende Strukturgleichungsmodell.

Abbildung 1: Vermutete Zusammenhänge der „Home Numeracy Environment“ mit der mathematischen Kompetenzentwicklung vom Kindergarten bis zum Ende der 1. Klasse unter Berücksichtigung der Intelligenz

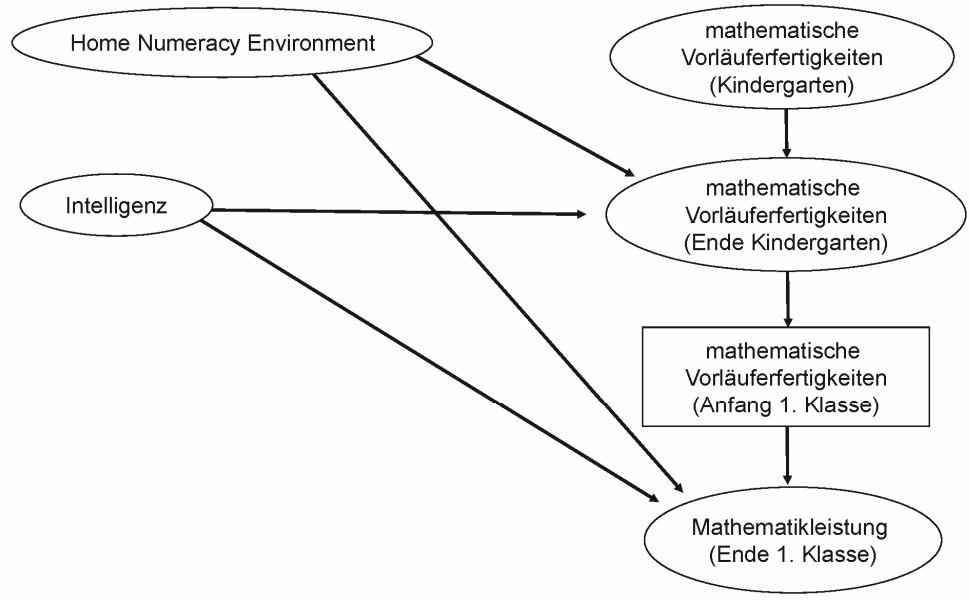




\section{Methode}

\section{Stichprobe und Datenerhebung}

Alle Erhebungen wurden im Rahmen der vom Ministerium für Kultus, Jugend und Sport des Landes Baden-Württemberg beauftragten wissenschaftlichen Begleitung des Projekts „Schulreifes Kind“ (SRK) ${ }^{1}$ durchgeführt (vgl. Hasselhorn et al. 2012). Die für die vorliegende Arbeit relevanten Einzeluntersuchungen im Kindergarten fanden im Frühjahr 2008 sowie im Sommer 2009 und damit eineinhalb Jahre sowie drei Monate vor der Einschulung statt. Insgesamt konnten im Kindergartenzeitraum von $N=609$ Kindern $(46,5 \%$ Mädchen, 53,5 \% Jungen) Informationen über ihre mathematische Lernumwelt erhoben werden. Diese Kinder waren zum Zeitpunkt der Einschulung zwischen 63 und 96 Monate alt. Das Altersmittel betrug bei der Einschulung $M=77$ Monate $(S D=4.5)$.

Zum Schuljahresanfang erfolgte eine weitere Einzelerhebung sowie am Schuljahresende zusätzlich eine Testung im Klassenverband. Alle Erhebungen fanden in den Kindergärten und Kindertagesstätten sowie in den Schulen verteilt auf mehrere Tage statt. Eine Einzeltestung nahm jeweils etwa 30 Minuten pro Kind in Anspruch und der Schulleistungstest Ende der 1. Klasse wurde innerhalb einer Schulstunde im Klassenverband durchgeführt. Geschulte Hilfskräfte, die neben einer ausführlichen Testeinweisung schriftliche Testleiterinformationen und ausführliche Instruktions- und Protokollbögen erhielten, übernahmen jeweils die Durchführung der Untersuchungen.

Aufgrund der Verteilung der Untersuchungen auf mehrere Tage konnte ein Teil der Kinder z.B. krankheits- oder urlaubsbedingt nicht mit allen Erhebungsverfahren untersucht werden, sodass im Kindergartenzeitraum nur $N=493$ Kinder an beiden Messzeitpunkten (MZP) untersucht werden konnten. Die Stichprobengröße reduzierte sich weiterhin beim Übertritt in die Schule, da nicht alle Schulen, in die die Kinder eingeschult wurden, in die weiteren Untersuchungen eingebunden werden konnten. Von einem Teil der Eltern lag zudem kein Einverständnis für die weitere Untersuchung vor. Insgesamt sind von $N=340$ Kindern Daten für die Schulzeit verfügbar.

\section{Erhebungsinstrumente}

Im Kindergarten wurde ein breites Spektrum an mathematischen Vorläuferfertigkeiten erfasst. Die beide Male im Kindergarten unverändert eingesetzte Testbatterie zur Erfassung mathematischer Kompetenzen (MZ-Test) wurde in leicht abgewandelter Form von Krajewski (2005; Krajewski/Schneider 2009) übernommen. In sechs Subtests wurden dabei Zählfertigkeiten, basale Rechenfertigkeiten, Ziffernkenntnis, Anzahlkonzept, Anzahlseriation

1 Wir danken dem Ministerium für Kultus, Jugend und Sport Baden-Württemberg für die Förderung der Untersuchung sowie allen beteiligten Untersuchungsleitern, den Kindern, Eltern, Erzieher(inn)en und Lehrer(inne)n für ihre engagierte Mitarbeit. Insbesondere gilt unser Dank unseren Kooperationspartnern bei der wissenschaftlichen Begleitung des Projekts „Schulreifes Kind“ in Würzburg (Sandra Schmiedeler, Robin Segerer), Heidelberg (Eva Biermeyer, Isabelle Keppler, Miriam Johnson, Herman Schöler) und Frankfurt (Katja Krebs, Hanna Wagner, Jan-Henning Ehm, Marcus Hasselhorn), ohne deren Beiträge zur Konzeption, Planung und Realisierung der Studie dieser Beitrag nicht hätte entstehen können. 
sowie Mengen schätzen und vergleichen untersucht, wobei insgesamt maximal 30 Punkte zu erreichen waren. Die interne Konsistenz des Gesamttests (Cronbachs $\alpha$ ) lag zum 1. MZP bei zufriedenstellenden $\alpha=.85$ und reduzierte sich zum 2. MZP auf noch ausreichende $\alpha=.75$.

Mittels der Columbia Mental Maturity Scale (CMM), die in der Basisdiagnostik für umschriebene Entwicklungsstörungen im Vorschulalter (BUEVA; Esser 2002) enthalten ist, wurde eine Einschätzung der Intelligenz zum 1. und 2. MZP vorgenommen. Hierbei sollten die Kinder aus vorgegebenen Zeichnungen (z.B. mehrere Gabeln) diejenige identifizieren, die nicht zu den übrigen passte (z.B. ein Löffel).

Kurz nach der Einschulung wurde die Lernausgangslage der Kinder mittels einer Computertestung erfasst. Mit dem hierbei eingesetzten Programm FIPS („Fähigkeitsindikatoren Primarschule“, basierend auf dem Programm „Performance Indicators in Primary Schools“ (PIPS); vgl. Bäuerlein et al. 2010) konnten u.a. die frühen Mathematikkenntnisse erhoben werden. Die Aufgaben umfassten Ziffernidentifikation, Identifikation von Flächen, mathematische Vorstellungen der Kinder und Rechenaufgaben. Sowohl die Retestreliabilität als auch die Validität sind als gut einzuschätzen (Bäuerlein et al. 2010; Tymms/Wylde 2004).

Weiterhin wurde zum Ende der 1. Klasse mit dem Deutschen Mathematiktest für 1. Klassen (DEMAT 1+; Krajewski et al. 2002) ein normierter und standardisierter Schulleistungstest eingesetzt.

\section{Erfassung der HNE}

Mittels eines Fragebogens wurden zum 2. MZP Informationen über die mathematische familiäre Lernumwelt („Home Numeracy Environment“, HNE) eingeholt. Hierzu sollten die Eltern drei Fragen beantworten, die auf die Häufigkeit von gemeinsamen mathematischen Aktivitäten mit ihren Kindern abzielten. Die Eltern wurden gefragt, wie häufig sie Würfelspiele (z.B. „Mensch ärgere Dich nicht“), Zählspiele (z.B. „Kosmolino: 1,2,3... Wie lerne ich die Zahlen“) oder Rechenspiele (z.B. „Ich lerne Rechnen“) mit ihrem Kind spielen. Die Items sollten auf einer fünfstufigen Skala beantwortet werden („,mehrmals wöchentlich“, „einmal wöchentlich“, ,alle 2-3 Wochen“, „seltener“, „nie“) und wurden zu einem Summenwert aufaddiert bzw. im Strukturgleichungsmodell als latentes Konstrukt modelliert. Daten konnten von $N=609$ Familien gesammelt werden. Die beobachteten Werte lagen zwischen 0 und 12 mit einem Mittelwert von $M=7.5(S D=3.1)$. Die interne Konsistenz (Cronbachs $\alpha$ ) lag bei zufriedenstellenden $\alpha=.76$.

\section{Statistische Auswertungen}

In einem ersten Auswertungsschritt wurden die Zusammenhänge aller Untersuchungsvariablen sowie zusätzlich von Geschlecht und Alter in einer Korrelationsmatrix dargestellt. Außerdem wurden die deskriptiven Statistiken aufgeführt.

In einem zweiten Auswertungsschritt wurden dann die gesamte Kompetenzentwicklung und dabei auch das Zusammenhangsmuster zwischen HNE, Intelligenz und den mathematischen Leistungen in einem Strukturgleichungsmodell (SEM) modelliert. Zur Datenimputation wurde bei der Erstellung des Strukturgleichungsmodells auf die „full in- 
formation maximum likelihood"-Methode zurückgegriffen, wobei das Analysemodell für alle Fälle, d. h. auch für diejenigen, die fehlende Werte aufwiesen, spezifiziert wurde. Dieses Vorgehen gilt derzeit als beste Möglichkeit für den Umgang mit fehlenden Werten (vgl. Lüdtke et al. 2007). Da zum 1. und 2. MZP das gleiche Testverfahren zur Erfassung mathematischer Vorläuferfertigkeiten eingesetzt wurde, wurden Messfehlerkorrelationen zwischen den MZP bei diesem Test im SEM zugelassen.

Das Grundgerüst des SEM stellt die mathematische Kompetenzentwicklung über alle MZP hinweg dar, wobei jede Leistung des vorhergehenden MZP die Leistung des darauffolgenden MZP vorhersagt. Zusätzlich wurde die Intelligenz als latente Variable, bestehend aus den beiden Intelligenzmessungen im Kindergarten, als Kontrollvariable zur Vorhersage der Mathematikleistung zum 2. MZP in das Modell aufgenommen, da Intelligenz einen engen Zusammenhang mit mathematischen Kompetenzen aufweist. Um zu überprüfen, ob HNE unter Kontrolle von mathematischem Vorwissen und Intelligenz einen Einfluss auf die Mathematikleistungen Ende der Kindergartenzeit ausübt, wurde ein entsprechender Pfad zwischen HNE und den mathematischen Vorläuferfertigkeiten zum 2. MZP in das SEM integriert. Darüber hinaus wurde auch die weitere mathematische Kompetenzentwicklung untersucht. Hierbei wurde mittels Pfaden von Intelligenz und HNE auf die Mathematikleistung Ende der 1. Klasse überprüft, ob HNE noch zusätzlichen Einfluss auf die mathematische Kompetenzentwicklung in der 1. Klasse unter Kontrolle der Intelligenz ausübt (vgl. Abb. 1). Im letztlich präsentierten Modell wurden jeweils nur signifikante Pfade integriert und Pfade bei fehlender Signifikanz entfernt. Als Kennwerte für die Modellgüte werden $X^{2} / \mathrm{df}$, IFI, TLI, CFI und RMSEA berichtet.

\section{Ergebnisse}

In Tabelle 1 werden die Mittelwerte und Standardabweichungen aller Variablen sowie die Korrelationen zwischen den Untersuchungsvariablen präsentiert.

Tabelle 1: Mittelwerte, Standardabweichungen und Korrelationen der Untersuchungsvariablen

\begin{tabular}{|c|c|c|c|c|c|c|c|c|c|}
\hline & & & & & & & & & \\
\hline & 1 & 2 & 3 & 4 & 5 & 6 & 7 & 8 & 9 \\
\hline (1) HNE & & .05 & -.06 & -.03 & .03 & .01 & $.09^{*}$ & $.12^{*}$ & $.15^{*}$ \\
\hline (2) Geschlecht + & & & .03 & -.02 & -.06 & .07 & .02 & $.28^{* \star}$ & $.13^{*}$ \\
\hline (3) Alter in Monaten bei Einschulung & & & & $.29^{* *}$ & $.13^{*}$ & $.33^{* *}$ & $.21^{* *}$ & $.12^{*}$ & -.01 \\
\hline (4) Intelligenz 1. MZP & & & & & $.40^{\star \star}$ & $.50^{\star \star}$ & $.42^{\star *}$ & $.39^{\star *}$ & $.39 * *$ \\
\hline (5) Intelligenz 2. MZP & & & & & & $.40^{* *}$ & $.41^{\star *}$ & $.30^{\star \star}$ & $.24^{* *}$ \\
\hline (6) Math. Vorläuferfertigkeiten 1. MZP & & & & & & & $.62^{\star \star}$ & $.62^{\star \star}$ & $.48^{* *}$ \\
\hline (7) Math. Vorläuferfertigkeiten 2. MZP & & & & & & & & $.61^{\star \star}$ & $.47^{* \star}$ \\
\hline (8) Math. Vorläuferfertigkeiten 3. MZP & & & & & & & & & $.54^{\star \star}$ \\
\hline (9) Mathematikleistung 4. MZP & & & & & & & & & \\
\hline Mittelwert $(M)$ & 7.50 & .54 & 77.02 & 46.32 & 52.27 & 17.73 & 25.80 & 26.59 & 23.81 \\
\hline Standardabweichung $(S D)$ & 3.12 & .50 & 4.51 & 7.59 & 3.95 & 7.27 & 4.00 & 7.69 & 7.49 \\
\hline
\end{tabular}


Es wird ersichtlich, dass die mathematischen Kompetenzen zu den verschiedenen MZP hohe und signifikante Zusammenhänge aufwiesen, obwohl im Untersuchungszeitraum drei unterschiedliche Testverfahren zur Erfassung der mathematischen Fähigkeiten herangezogen wurden. Für HNE zeigten sich keine Zusammenhänge mit den Kontrollvariablen und signifikante Korrelationen mit der Mathematikleistung erst ab dem 2. MZP, wobei diese Zusammenhänge als eher gering einzustufen sind (kleine Effekte). Für den Zusammenhang von Geschlecht und Mathematikkompetenz konnten erst ab Schulbeginn (3. MZP) signifikante Korrelationen beobachtet werden, wobei Jungen höhere Leistungen aufwiesen. Das Alter der Kinder hing nur für die ersten drei MZP signifikant mit den mathematischen Leistungen zusammen, nicht jedoch am Ende des ersten Schuljahres. Die Intelligenz korrelierte mit den mathematischen Leistungen während des gesamten Untersuchungszeitraums moderat bis hoch.

Die korrelativen Zusammenhänge basieren auf der durch den Dropout reduzierten Stichprobe und berücksichtigen außerdem nicht die Interaktionen zwischen den verschiedenen Variablen. Deshalb wurden die mathematische Kompetenzentwicklung und der Einfluss von HNE und Intelligenz darauf mittels eines Strukturgleichungsmodells überprüft (vgl. Abb. 2).

Abbildung 2: Mathematische Kompetenzentwicklung vom Kindergarten bis zum Ende der 1. Klasse unter Berücksichtigung von Intelligenz und „Home Numeracy Environment"

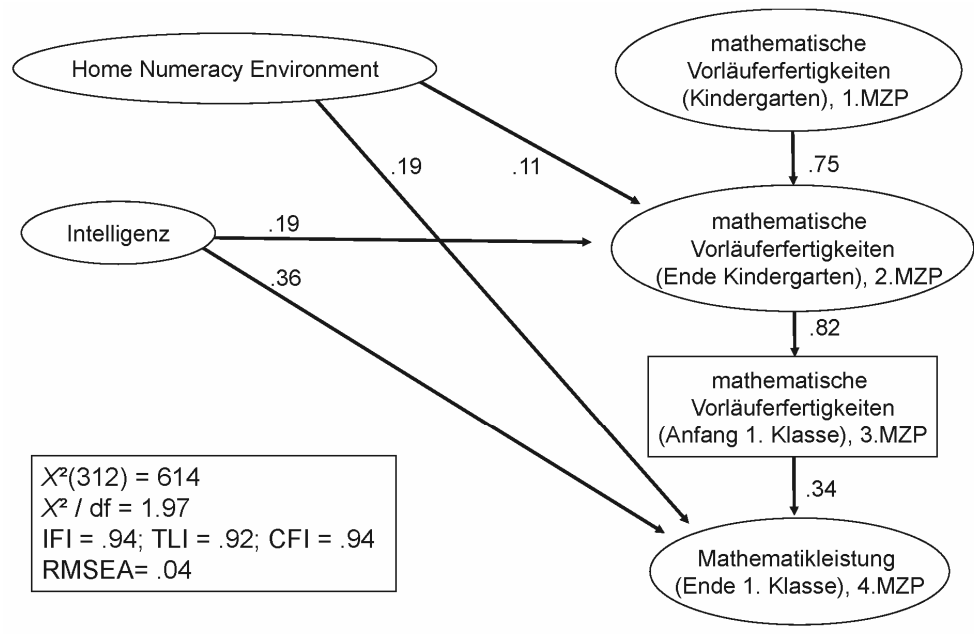

Alle vermuteten Pfade wurden signifikant, weshalb für das Endmodell keine Pfade entfernt werden mussten. Die Kennwerte für die Modellgüte sprechen für eine sehr gute Passung zwischen dem Modell und den Daten (vgl. Weiber/Mühlhaus 2010).

Auf der rechten Seite der Abbildung ist die mathematische Kompetenzentwicklung von MZP 1 (Kindergarten) bis MZP 4 (Ende 1. Klasse) dargestellt. Wie sich durch die Korrelationen schon angedeutet hat, war die Vorhersageleistung der mathematischen Leistungen vom früheren MZP auf den nachfolgenden MZP sehr hoch und nahm nur für die Vorhersage des 4. MZP durch den 3. MZP etwas ab. Die Intelligenz als Kontrollvari- 
able hatte sowohl Einfluss auf die Vorläuferfertigkeiten zum Ende der Kindergartenzeit als auch zusätzlich auf die Mathematikleistung am Ende der 1. Klasse. Auf die Integration eines zusätzlichen direkten Pfades von Intelligenz auf die mathematischen Vorläuferfertigkeiten zum MZP 1 wurde verzichtet, da dieser - in der Überprüfung sehr enge - Zusammenhang nicht direkt unsere Fragestellungen betrifft. Die Modellgüte sowie die Bedeutung von HNE für die mathematische Kompetenzentwicklung blieben davon jedoch unbeeinträchtigt.

So gilt auch für die zentrale Untersuchungsvariable HNE das Gleiche wie für die Intelligenz. HNE beeinflusste nicht nur die mathematischen Fähigkeiten Ende des Kindergartens signifikant, sondern darüber hinaus auch die weitere mathematische Kompetenzentwicklung in der 1. Klasse. Zwar gelang durch HNE allein nur eine geringe Varianzaufklärung der mathematischen Leistungen zum 4. MZP (knapp 5\%). Allerdings muss berücksichtigt werden, dass hierbei sowohl mathematisches Vorwissen als auch die Intelligenz der Kinder kontrolliert wurden.

\section{Diskussion}

Die vorliegende Studie hatte zum Ziel, den Einfluss der familiären Lernumwelt auf die mathematische Kompetenzentwicklung zu analysieren. Auch wenn schon seit langem bekannt ist, dass die Familie eine wichtige Rolle spielt (z.B. Resnick 1989), gibt es bislang kaum Untersuchungen zur „Home Numeracy Environment“ und deren Bedeutung für die mathematischen Fähigkeiten von Kindern, die in dieser Lernumwelt leben. Als wesentlicher Befund der Analysen kann angesehen werden, dass HNE auch nach Kontrolle mathematischer Vorkenntnisse und der Intelligenz der Kinder nicht nur die mathematischen Vorläuferfertigkeiten zum Ende des Kindergartens, sondern auch zusätzlich die Mathematikleistung am Ende der 1. Klasse bedeutsam vorhersagen konnte (vgl. auch LeFevre et al. 2009).

Daneben spielte aber auch die Intelligenz eine sehr wichtige Rolle für die Mathematikentwicklung. Mit Blick auf die Literatur ist die Befundlage hierzu jedoch nicht ganz eindeutig. Während beispielsweise Hasselhorn und Gold (2009) sowie Krajewski und Schneider (2009) die besondere Bedeutung von Intelligenz für mathematische Kompetenzen betonen, verweisen Stern und Kollegen (2006) darauf, dass die Intelligenz weniger genaue Vorhersagen der Mathematikleistung ermöglicht als spezifisches mathematisches Vorwissen. Die Befunde aus dieser Studie unterstützen eher die erste Sichtweise, wobei abzuwarten gilt, wie sich die Zusammenhänge für die weitere Kompetenzentwicklung und damit spätere MZP darstellen werden.

Obwohl die mathematischen Fähigkeiten durch HNE signifikant vorhergesagt werden konnten, waren die Effektgrößen und die Varianzaufklärung eher gering. Dies war mit Blick auf frühere Untersuchungen zu HLE so zu erwarten (z.B. Scarborough/Dobrich 1994). Dennoch sind auch solch kleine Effekte bedeutsam, da sie über die Zeit kumulieren können, und da die familiäre Lernumwelt sich leichter beeinflussen lässt als beispielsweise die Intelligenz oder der familiäre Hintergrund.

HNE wurde in der vorliegenden Studie aufgrund der großen Stichprobe mittels eines Fragebogens erhoben. Obwohl dabei das Risiko sozial erwünschter Antworten auf die ge- 
stellten Fragen nicht ausgeschlossen werden kann, zeigten frühere Studien zu HLE, dass diese Form der Erhebung zu zuverlässigen Daten führt und eine große Übereinstimmung mit anderen Erhebungsverfahren gegeben ist (vgl. Burgess 2002). Weiterhin weist der exakt im Durchschnitt der Skala liegende Mittelwert für HNE in unserer Stichprobe auf keine Verzerrung der Daten hin. Dennoch wäre es für zukünftige Studien wünschenswert, die Anzahl der Items in der Skala deutlich aufzustocken, um die Reliabilität weiter zu verbessern.

Da die Eltern nur einmal zur HNE befragt werden konnten, war es nicht möglich, die Stabilität der von den Eltern gebotenen Lernumwelt zu bestimmen. Deshalb kann nicht ausgeschlossen werden, dass es im Verlauf der Untersuchung in manchen Familien bedeutsame Veränderungen bei der Häufigkeit mathematischer Aktivitäten gab. Allerdings deutet die Forschung zu sowohl HLE als auch HNE auf hohe Stabilitäten der familiären Lernumwelt im Vorschul- und frühen Grundschulalter hin (LeFevre et al. 2009; Niklas 2011).

Als Indikator für HNE wurde in der vorliegenden Studie der Summenscore dreier Items herangezogen. Während die Häufigkeit, mit der Würfelspiele in der Familie gespielt werden, eher der indirekten mathematischen Lernumwelt zugerechnet werden kann, beziehen sich Zähl- und Rechenspiele eher auf die direkte, formelle mathematische Lernumwelt (vgl. LeFevre et al. 2009; LeFevre et al. 2010). Insbesondere die informelle Lernumwelt und damit die Häufigkeit, mit der Würfelspiele gespielt werden, scheint eine wichtige Rolle in der mathematischen Kompetenzentwicklung einzunehmen (für dieses Item wurden auch die höchsten Korrelationen mit den mathematischen Fähigkeiten zum 2. MZP bis zum 4. MZP gefunden mit $r=.15$ bis .18; $p<.01$ ). Auch andere Studien weisen darauf hin, dass Würfelspiele den Erwerb mathematischen Wissens unterstützen können (Ramani/Siegler 2008). Erklärt werden kann dieser Befund damit, dass informelles Lernen eher häufiger als formelles Lernen in den Familien stattfindet, und dass junge Kinder für indirektes Lernen durchaus empfänglich sind. Da in der vorliegenden Studie aber nur drei Items zur Erfassung der HNE eingesetzt wurden, gilt es in zukünftigen Arbeiten, die Beziehung formeller und informeller Aspekte der HNE mit mathematischen Kompetenzen genauer zu untersuchen.

Eine weitere Überprüfung der bei uns gefundenen Zusammenhänge erscheint auch bezüglich unserer Testauswahl zur Erfassung mathematischer Kompetenzen sinnvoll. Zwar fanden sich hohe Zusammenhänge zwischen den insgesamt drei verschiedenen mathematischen Testverfahren, die in unserer Untersuchung eingesetzt wurden, da aber hierbei sowohl zwischen ausgedruckten Tests und Computertests als auch zwischen Einzelerhebungen und Gruppenerhebungen variiert wurde, könnten die Ergebnisse hierdurch beeinflusst worden sein.

Es ergeben sich einige praktische Implikationen aus den vorliegenden Befunden. So wird ersichtlich, dass über eine Veränderung der familiären Lernumwelt auch die mathematischen Kompetenzen der in ihr lebenden Kinder in einem gewissen Umfang beeinflusst werden können. Dies kann einerseits indirekt geschehen, indem die mathematischen Kompetenzen und Einstellungen der Eltern gestärkt werden (vgl. Blevins-Knabe et al. 2000; Ehmke/Siegle 2008). So konnten beispielsweise Ehmke und Siegle (2008) bei Jugendlichen zeigen, dass eine höhere mathematische Kompetenz ihrer Eltern auch mit vermehrten kulturellen Aktivitäten, mehr Lernunterstützung und mehr Kulturgütern im Haushalt einherging. Diese Umstände führten dann letztlich auch zu besseren Mathematikleistungen der Jugendlichen. 
Wesentlich einfacher scheint jedoch der direkte Weg über gemeinsame mathematische Aktivitäten von Eltern mit ihren Kindern wie in der vorliegenden Studie zu sein. So sind auch nach Ramey und Ramey (1998) Interventionen für Kinder mit Entwicklungsdefiziten sinnvoll, die sich direkt auf diese Kinder beziehen und dabei möglichst intensiv durchgeführt werden. Im Vergleich zu anderen Betreuungspersonen ihrer Kinder können insbesondere Eltern sehr förderlich auf die kindliche Entwicklung einwirken, da sie eher die Möglichkeit haben, die dafür erforderliche Zeit aufzubringen. Eltern sind sich dieser Rolle auch durchaus bewusst und sehen sich in verschiedenen Bereichen wie z.B. bei der Sprachentwicklung und dem Erwerb mathematischer Fähigkeiten als die Haupteinflusskraft für das Lernen ihrer Kinder an (Musun-Miller/Blevins-Knabe 1998).

Natürlich sind aber nicht nur die Eltern gefordert, sondern auch in Kindergärten können mathematische Vorläuferfertigkeiten gefördert werden. Zunehmend wird in den Bildungs- und Erziehungsplänen der letzten Jahre hierauf auch Bezug genommen (z.B. Ministerium für Kultus, Jugend und Sport Baden-Württemberg 2009). Auch hier bieten die vorliegende Befunde Anhaltspunkte, wie Kinder spielerisch und sinnvoll in ihrer mathematischen Entwicklung unterstützt werden können.

Wie die Ergebnisse der vorliegenden Studie zeigen, müssen die durchgeführten Aktivitäten nicht standardisierte oder komplexe Programme sein. Vielmehr können schon einfache mathematische Aktivitäten in einem spielerischen Kontext einen bedeutenden Beitrag zur mathematischen Kompetenzentwicklung leisten, wenn sie nur häufig genug durchgeführt werden. So greifen auch einzelne Förderprogramme zur Verbesserung mathematischer Fähigkeiten auf spielerische Elemente zurück (vgl. Werner 2009).

Die förderlichen Effekte mathematischer Spiele lassen sich über deren Spielstruktur erklären. So werden beispielsweise bei vielen Würfelspielen einfache Additionen und Zählleistungen in regelhafter und sich wiederholender Weise eingeübt, während Zählspiele oft Kenntnisse über Zahlwörter und Ziffernsymbole vermitteln. Auch Naegele (2001) verweist darauf, dass Würfelspiele eine gute Vorbereitung für den Umgang mit Zahlen darstellen, bevor das eigentliche Rechnen in der Schule vermittelt wird. Dabei können auch sehr einfache Würfel- und Zahlenspiele wichtige Mittel sein, um komplexere mathematische Strukturen wie z.B. das Zahlensystem oder den mentalen Zahlenstrahl zumindest leichter zu erlernen und zu verstehen (vgl. Ramani/Siegler 2008; Siegler/Ramani 2009). Letztlich bieten mathematische Spiele den Vorteil, dass spielerische Übungen Kindern zumeist mehr Freude bereiten und eher Motivation entstehen lassen als formelles Lernen und dabei gleichzeitig Müdigkeit, Aggressionen oder Unkonzentriertheit eher seltener auftreten (Naegele 2001).

\section{Literatur}

Bäuerlein, K., Faust, G., Franz, U., Jost, M. \& Schneider, W. (2010). Fähigkeitsindikatoren Primarschule (FIPS) - Erprobung und Weiterentwicklung einer internationalen computerbasierten Schulanfangsdiagnose. In: Hasselhorn, M. \& Schneider, W. (Hrsg.), Frühprognose schulischer Kompetenzen. Tests und Trends. Göttingen: Hogrefe, S. 85-108 (N. F., Band 9).

Blevins-Knabe, B., Berghout Austin, A., Musun L., Eddy, A. \& Jones, R. M. (2000). Family home care providers' and parents' beliefs and practices concerning mathematics with young children. Early Child Development and Care, 165, 1, S. 41-58. 
Blevins-Knabe, B. \& Musun-Miller, L. (1996). Number use at home by children and their parents and its relationship to early mathematical performance. Early Development and Parenting, 5, 1, S. 35-45.

Brunner, E. J. \& Noack, P. (2010). Familieninteraktion/Familienerziehung. In: Rost, D. H. (Hrsg.), Handwörterbuch Pädagogische Psychologie. Weinheim: Beltz, S. 190-197 (4. Auflage).

Burgess, S. R. (2002). The influence of speech perception, oral language ability, the home literacy environment, and pre-reading knowledge on the growth of phonological sensitivity: A one-year longitudinal investigation. Reading and Writing, 15, S. 709-737.

Butterworth, B. (2005). The development of arithmetical abilities. Journal of Child Psychology and Psychiatry, 46, 1, S. 3-18.

Christian, K., Morrison, F. J. \& Bryant, F. B. (1998). Predicting kindergarten academic skills: Interactions among child care, maternal education, and family literacy environments. Early Childhood Research Quarterly, 13, 3, S. 501-521.

Claessens, A., Duncan, G. \& Engel, M. (2009). Kindergarten skills and fifth-grade achievement: Evidence from the ECLS-K. Economics of Education Review, 28, S. 415-427.

Duncan, G. J., Dowsett, C. J., Claessens, A., Magnuson, K., Huston, A. C., Klebanov, P., Pagani, L. S., Feinstein, L., Engel, M., Brooks-Gunn, J., Sexton, H., Duckworth, K. \& Japel, C. (2007). School readiness and later achievement. Developmental Psychology, 43, 6, S. 1428-1446.

Ehmke, T. \& Siegle, T. (2008). Einfluss elterlicher Mathematikkompetenz und familialer Prozesse auf den Kompetenzerwerb von Kindern in Mathematik. Psychologie in Erziehung und Unterricht, 55, S. 253-264.

Esser, G. (2002). BUEVA - Basisdiagnostik für umschriebene Entwicklungsstörungen im Vorschulalter. Göttingen: Beltz.

Geary, D. C. (2000). From infancy to adulthood: the development of numerical abilities. European Child \& Adolescent Psychiatry, 9, 2, S. II/11-II/16.

Geary, D. C. (2011). Cognitive predictors of achievement growth in mathematics: A 5-year longitudinal study. Developmental Psychology, 47, 6, S. 1539-1552.

Hasselhorn, M. \& Gold, A. (2009). Pädagogische Psychologie. Erfolgreiches Lernen und Lehren. Stuttgart: Kohlhammer (2. durchgesehene Auflage).

Hasselhorn, M., Schöler, H., Schneider, W., Ehm, J.-H., Johnson, M., Keppler, I., Krebs, K., Niklas, F., Randhawa, E., Schmiedeler, S., Segerer, R. \& Wagner, H. (2012). Gezielte Zusatzförderung im Modellprojekt „Schulreifes Kind“ - Auswirkungen auf Schulbereitschaft und schulischen Lernerfolg. Frühe Bildung, 1, 1, S. 3-10.

Helmke, A. \& Schrader, F.-W. (2010). Determinanten der Schulleistung. In: Rost, D. H. (Hrsg.), Handwörterbuch Pädagogische Psychologie. Weinheim: Beltz, S. 90-102 (4. Auflage).

Hood, M., Conlon, E. \& Andrews, G. (2008). Preschool home literacy practices and children's literacy development: A longitudinal analysis. Journal of Educational Psychology, 100, 2, S. 252-271.

Huntsinger, C. S., Jose, P. E., Larson, S. L., Balsink Krieg, D. \& Shaligram, C. (2000). Mathematics, vocabulary, and reading development in Chinese-American and European-American children over the primary school years. Journal of Educational Psychology, 92, S.745-760.

Hyde, J. S., Else-Quest, N. M., Alibali, M. W., Knuth, E. \& Romberg, T. (2006). Mathematics in the home: Homework practices and mother-child interactions doing mathematics. The Journal of Mathematical Behavior, 25, S.136-152.

Krajewski, K. (2005). Vorschulische Mengenbewusstheit von Zahlen und ihre Bedeutung für die Früherkennung von Rechenschwäche. In: Hasselhorn, M., Marx, H. \& Schneider, W. (Hrsg.), Diagnostik von Mathematikleistungen. Test und Trends. Göttingen: Hogrefe, S. 49-70 (N. F., Band 4).

Krajewski, K., Küspert, P., Schneider, W. \& Visé, M. (2002). Deutscher Mathematiktest für erste Klassen (DEMAT 1+). Göttingen: Hogrefe.

Krajewski, K. \& Schneider, W. (2009). Early development of quantity to number-word linkage as a precursor of mathematical school achievement and mathematical difficulties: Findings from a four-year longitudinal study. Learning and Instruction, 19, S. 513-526.

LeFevre, J., Clarke, T. \& Stringer, A. P. (2002). Influences of language and parental involvement on the development of counting skills: Comparisons of French- and English-speaking Canadian children. Early Child Development and Care, 172, S. 283-300. 
LeFevre, J.-A., Polyzoi, E., Skwarchuk, S.-L., Fast, L. \& Sowinski, C. (2010). Do home numeracy and literacy practices of Greek and Canadian parents predict the numeracy skills of kindergarten children? International Journal of Early Years Education, 18, 1, S. 55-70.

LeFevre, J.-O., Skwarchuk, S.-L., Smith-Chant, B. L., Fast, L., Kamawar, D. \& Bisanz, J. (2009). Home numeracy experiences and children's math performance in the early school years. Canadian Journal of Behavioural Science, 41, 2, S. 55-66.

Lüdtke, O., Robitzsch, A., Trautwein, U. \& Köller, O. (2007). Umgang mit fehlenden Werten in der psychologischen Forschung. Probleme und Lösungen. Psychologische Rundschau, 58, S. 103-117.

Melhuish, E. C., Phan, M. B., Sylva, K., Sammons, P., Siraj-Blatchford, I. \& Taggart, B. (2008). Effects of the home learning environment and preschool centre experience upon literacy and numeracy development in early primary school. Journal of Social Issues, 64, S. 95-114.

Ministerium für Kultus, Jugend und Sport Baden-Württemberg (2009). Orientierungsplan für Bildung und Erziehung in baden-württembergischen Kindergärten und weiteren Kindertageseinrichtungen (Vorl. Fassung nach Anhörung vom 17.06.2009). Stuttgart: Ministerium für Kultus, Jugend und Sport (Unveröffentliches Dokument).

Musun-Miller, L. \& Blevins-Knabe, B. (1998). Adults' beliefs about children and mathematics: How important is it and how do children learn about it? Early Development and Parenting, 7, S. 191-202.

Naegele, I. M. (2001). Schulschwierigkeiten in Lesen, Rechtschreibung und Rechnen. Vorbeugen, verstehen, helfen. Ein Elternhandbuch. Weinheim: Beltz.

Niklas, F. (2011). Vorläuferfertigkeiten im Vorschulalter zur Vorhersage der Schulfähigkeit, späterer Rechenschwäche und Lese- und Rechtschreibschwäche. Diagnostik, Zusammenhänge und Entwicklung in Anbetracht der bevorstehenden Einschulung. Hamburg: Dr. Kovač.

Niklas, F. \& Schneider, W. (2010). Der Zusammenhang von familiärer Lernumwelt mit schulrelevanten Kompetenzen im Vorschulalter. Zeitschrift für Soziologie der Erziehung und Sozialisation, 30, 2, S. 148-164.

Ramani, G. \& Siegler, R. S. (2008). Promoting broad and stable improvements in low-income children's numerical knowledge through playing number board games. Child Development, 29, S. 375-394.

Ramey, C. T. \& Ramey, S. L. (1998). Early intervention and early experience. American Psychologist, 53,2, S.109-120.

Rashid, F. L., Morris, R. D. \& Sevcik, R. A. (2005). Relationship between Home Literacy Environment and reading achievement in children with reading disabilities. Journal of Learning Disabilities, 38, 1, S. 2-11.

Resnick, L. B. (1989). Developing mathematical knowledge. American Psychologist, 44, 2, S. 162-169.

Roßbach, H.-G. (2005). Effekte qualitativ guter Betreuung, Bildung und Erziehung im frühen Kindesalter auf Kinder und ihre Familien. In: Ahnert, L., Roßbach, H.-G., Neumann, U., Heinrich, J. \& Koletzko, B. (Hrsg.), Bildung, Betreuung und Erziehung von Kindern unter sechs Jahren. Wiesbaden: VS Verlag für Sozialwissenschaften, S. 55-174.

Scarborough, H. S. \& Dobrich, W. (1994). On the efficacy of reading to preschoolers. Developmental Review, 14, S. 245-302.

Siegler, R. S. \& Ramani, G. B. (2009). Playing linear number board games - but not circular ones - improves low-income preschoolers' numerical understanding. Journal of Educational Psychology, 101, S. 545-560.

Skwarchuk, S.-L. (2009). How do parents support preschoolers' numeracy learning experiences at home? Early Childhood Education Journal, 37, S. 189-197.

Stern, E. (2006). Wie viel Hirn braucht die Schule? Chancen und Grenzen einer neuropsychologischen Lehr-Lern-Forschung. In: Caspary, R. (Hrsg.), Lernen und Gehirn. Der Weg zu einer neuen Pädagogik. Freiburg im Breisgau: Herder, S. 128-141.

Stern, E. (2008). Verpasste Chancen? Was wir aus der LOGIK-Studie über den Mathematikunterricht lernen können. In: Schneider, W. (Hrsg.), Entwicklung von der Kindheit bis zum Erwachsenenalter. Befunde der Münchner Längsschnittstudie LOGIK. Weinheim: Beltz, S. 187-202.

Stern, E. (2009). Development of mathematical competencies: Source of individual differences and their developmental trajectories. In: Schneider, W. \& Bullock, M. (Hrsg.), Human development from ear- 
ly childhood to early adulthood. Findings from a 20 year longitudinal study. New York: Psychology Press, S. 221-238.

Stern, E., Felbrich, A. \& Schneider, M. (2006). Mathematiklernen. In: Rost, D. H. (Hrsg.), Handwörterbuch Pädagogische Psychologie. Weinheim: Beltz, 461-469 (3. überarbeitete und erweiterte Auflage).

Tymms, P. \& Wylde, M. (2004). Basisprüfverfahren und Dauerbeobachtung in der Grundschule. In: Faust, G., Götz, M., Hacker, H. \& Roßbach, H.-G. (Hrsg.), Anschlussfähige Bildungsprozesse im Elementar- und Primarbereich. Bad Heilbrunn: Klinkhardt, S. 190-203.

Walston, J. \& West, J. (2004). Full-day and half-day kindergarten in the United States: Findings from the Early Childhood Longitudinal Study, Kindergarten Class of 1998-99. Washington, DC: U.S. Government Printing Office.

Weiber, R. \& Mühlhaus, D. (2010). Strukturgleichungsmodellierung. Eine anwendungsorientierte Einführung in die Kausalanalyse mit Hilfe von AMOS, SmartPLS und SPSS. Heidelberg: Springer.

Werner, B. (2009). Dyskalkulie - Rechenschwierigkeiten. Diagnose und Förderung rechenschwacher Kinder an Grund- und Sonderschulen. Stuttgart: Kohlhammer.

Whitehead, M. (2004). Sprachliche Bildung und Schriftsprachkompetenz (literacy) in der frühen Kindheit. In: Fthenakis, W. E. \& Oberhuemer, P. (Hrsg.), Frühpädagogik international - Bildungsqualität im Blickpunkt. Wiesbaden: VS Verlag für Sozialwissenschaften, S. 295-311.

Eingereicht am/Submitted on: 14.03.2012

Angenommen am/Accepted on: 06.07.2012

Anschriften der Autoren/Addresses of the authors:

Dr. Frank Niklas (Korrespondenzautor/Corresponding author)

Prof. Dr. Wolfgang Schneider

Universität Würzburg

Lehrstuhl für Psychologie IV

Röntgenring 10

97070 Würzburg

Deutschland/Germany

E-Mail: niklas@psychologie.uni-wuerzburg.de schneider@psychologi.uni-wuerzburg.de 